\title{
Limiting Consumption in Tuberculosis: Current Concepts in anti- Tuberculosis Treatment in the Critically Ill Patient
}

\author{
Mervyn Mer, FRCP, $\mathrm{PhD}^{1}$; Alimudin Zumla, FRCP, $\mathrm{PhD}^{2}$; \\ Martin W. Dünser, MD ${ }^{3}$
}

\begin{abstract}
1, Department of Medicine, Divisions of Critical Care and Pulmonology, Charlotte Maxeke Johannesburg Academic Hospital and Faculty of Health Sciences University of Witwatersrand, Johannesburg, South Africa.

2, Division of Infection and Immunity, University College London Medical School, and NIHR Biomedical Research Center at University College of London Hospitals, London, United Kingdom;

3, Department of Anesthesiology and Intensive Care Medicine, Kepler University Hospital, Johannes Kepler University Linz, Linz, Austria.
\end{abstract}

Short running title: Anti-TB therapy in the critically ill

Word count: 998 words

None of the authors has a conflict of interest in regards of drugs, methods or techniques discussed in this manuscript. 
Once considered a waning disease, the burden of tuberculosis (TB) has increased around the world over the last decades. Today, TB is the leading cause of mortality associated with a single infectious pathogen [1]. Although treatment regimens as suggested by the World Health Organization (WHO) result in survival rates of 95\% under trial conditions [2], the mortality of TBassociated critical illness remains unacceptably high, and exceeds $50 \%$ in many cohorts [3]. This makes Mycobacterium tuberuclosis one of the deadliest causes of sepsis, a fact often not recognized by many clinicians in high-income countries. The exponential increase in mortality between noncritically and critically ill patients with active TB disease suggests that antiTB treatment regimens may be inadequate once organ dysfunction and sepsis have occurred. This concise review focuses on novel pharmacokinetic considerations and suggests a potential novel approach to anti-TB treatment in the critically ill patient.

\section{Who should be treated?}

Timely initiation of anti-TB therapy is essential for treatment success, particularly in patients with a high disease severity [3]. This crucially depends on early recognition of at-risk populations and rapid confirmation of the diagnosis. Risk factors for active TB disease broadly include poor living conditions, previous infection or contact with TB (e.g. people living in or originating from endemic regions, prisoners, healthcare workers), immunosuppression (e.g. human immunodeficiency virus (HIV) infection, immunosuppressive therapy including biologics, alcoholism), extremes of age, and medical conditions known to increase the risk for TB (e.g. COPD, 
silicosis, diabetes mellitus, malnutrition). Acute respiratory failure, altered mental state, septic shock and multiple organ dysfunction are the most common causes of critical illness and intensive care unit admission of patients with TB. Rapid TB tests based on nucleic acid amplification techniques (e.g. GeneXpert MTB/RIF) are sensitive and specific to diagnose active TB disease in both HIV-negative and -positive patients within a few hours [4]. Simultaneously, these tests can identify multi-drug resistant (MDR) strains.

\section{What anti-TB treatment regimen should be started in critically ill patients?}

For both adults and children, the WHO recommends an intensive treatment regimen (rifampicin+isoniazid+ethambutol+pyrazinamide) for two months, followed by a continuation regimen (rifampicin+isoniazid) for four to seven months [5]. Treatment regimens for MDR TB include at least four second line anti-TB drugs [5].

\section{Pharmacokinetic considerations in critically ill patients}

Although only limited data on pharmacokinetic changes of anti-TB drugs in critically ill patients have been published, those available consistently report inadequate anti-TB drug levels. In a South African population, therapeutic drug levels were detected in $<30 \%$ of critically ill patients when a standard drug regimen was given as a fixed drug combination dose via a nasogastric tube [6]. Multiple factors alter pharmacokinetics during critical illness [7]. 
Intestinal drug absorption is commonly delayed or altered by gastroparesis, intestinal paralysis, pharmacological ulcer prophylaxis, and changes in the gut microbiome. Fluid accumulation increases the volume of distribution of anti-TB drugs with consequent reduction in serum and tissue levels. In addition, glomerular hyperfiltration [7] resulting in augmented renal clearance of anti-TB drugs, genetic variations in drug metabolization (e.g. isoniazid), and poor penetration into infected compartments [8] are likely to contribute to sub-therapeutic levels at the site of TB infection. Such subtherapeutic anti-TB drug levels have been associated with treatment failure and increased mortality $[9,10]$.

\section{A novel approach to anti-TB treatment in critically ill patients}

In view of the aforementioned pharmacokinetic changes, it appears pragmatic to modify the initial anti-TB treatment in critically ill patients to optimize and achieve therapeutic blood levels of anti-TB drugs (Figure 1). During the phase of critical illness, anti-TB drugs should be administered intravenously and probably at higher dosages than recommended by current guidelines. Although rifampicin, the drug with the highest sterilizing TB activity, is available in an intravenous formulation, it and other standard anti-TB drugs are not universally available in this form. Consequently, modified regimens including intravenous formulations of alternative and highly effective anti-TB drugs (e.g. rifampicin+moxifloxacin+amikacin) may be administered. Empirical intravenous fluoroquinolone use improved the survival of critically ill patients admitted for pulmonary TB [11]. Several studies have reported that higher than usual doses of anti-TB drugs (e.g. 
rifampicin up to $35 \mathrm{mg} / \mathrm{kg} / \mathrm{d}$ [12]) were safe, reduced the time to culture conversion in liquid media, and may be associated with better survival in patients with severe disease $[12,13]$. A recent large trial evaluating an intensified regimen including higher-dose rifampicin $(15 \mathrm{mg} / \mathrm{kg} / \mathrm{d})$ and levofloxacin $(20 \mathrm{mg} / \mathrm{kg} / \mathrm{d})$ for the first eight weeks, however, failed to confirm these results in adults with TB meningitis [14]. Since only $17.4 \%$ of study patients presented with a high disease severity, the implications of this trial for the management of critically ill patients remain unclear. The use of therapeutic drug monitoring (TDM) has also been advocated to optimize dosing of anti-TB drugs relative to minimum inhibitory concentrations (MICs) [15]. Critical drawbacks of such a strategy are the restricted availability of this useful adjunct in many endemic regions, and the fact that MICs are only known after culture and resistance testing. In all critically ill patients and especially in those with MDR TB disease, infectious disease specialist should assist with the empirical selection and dosing of anti-TB drugs, as well as their interactions with other drugs (e.g. HIV therapy). During recovery from critical illness, high-dose intravenous anti-TB therapy can be switched to currently recommended enteral, fixed dose regimens (Figure 1). The time point for this transition is clinically highlighted and determined by improvement of organ function, in particular gastrointestinal function (e.g. full tolerance of enteral feeds), and reversal of fluid accumulation.

\section{Conclusions}


TB remains an enormous healthcare problem with a billion lives having been consumed over the last two centuries. Scores of critically ill patients with TB are encountered globally on a daily basis. It is incumbent on intensive care practitioners to be aware of the problem and focus on efforts to address the unacceptably poor outcomes associated with this disease entity. Although various factors may influence, optimizing therapeutic approaches may very well limit "consumption" in critically ill patients with TB. We eagerly and excitedly await further work in this sphere! 


\section{REFERENCES}

1. World Health Organization. Tuberculosis Factsheet. March 2017. http:/ / www.who.int/mediacentre/factsheets/fs 104/en/ [accessed Oct 22, 2017].

2. Zumla A, Raviglione M, Hafner R, von Reyn F (2013) Tuberculosis. N Engl $\mathrm{J}$ Med 368:745-755.

3. Kethireddy S, Light RB, Mirzanejad Y, Maki D, Arabi Y, Lapinsky S, Sion D, Kumar A, Parrillo JE, Kumar A; Cooperative Antimicrobial Therapy of Septic Shock (CATSS) Database Group (2013). Mycobacterium tuberculosis septic shock. Chest 144:474-482.

4. Steingart KR, Schiller I, Horne DJ, Pai M, Boehme CC, Dendukuri N (2014) Xpert® MTB/RIF assay for pulmonary tuberculosis and rifampicin resistance in adults. Cochrane Database Syst Rev CD009593.

5. World Health Organization. Guidelines for treatment of tuberculosis. Fourth edition. 2010; WHO/HTM/TB/2009.420.

6. Koegelenberg CF, Nortje A, Lalla U, Enslin A, Irusen EM, Rosenkranz B, Seifart HI, Bolliger CT (2013) The pharmacokinetics of enteral anti-TB drugs in patients requiring intensive care. S Afr Med J 103:394-398.

7. Mer M, Lipman (2015) Antibiotic administration in the critically ill - in need of intensive care! S Afr Med J 105(5): 357-9

8. Shenje J, Ifeoma Adimora-Nweke F, Ross IL, Ntsekhe M, Wiesner L, Deffur A, McIlleron HM, Pasipandoya J, Gumbo T, Mayosi BM (2015) Poor penetration of antibiotics into pericardium in pericardial tuberculosis. EBioMedicine 2:1640-1649.

9. Swaminathan S, Pasipanodya JG, Ramachandran G, Hemanth Kumar AK, Srivastava S, Deshpande D, Nuermberger E, Gumbo T (2016) Drug concentration thresholds predictive of therapy failure and death in children with tuberculosis: bread crumb trails in random forests. Clin Infect Dis 63(Suppl 3):S63-S74.

10. Te Brake L, Dian S, Ganiem AR, Ruesen C, Burger D, Donders R, Ruslami R, van Crevel R, Aarnoutse R (2015)

Pharmacokinetic/pharmacodynamics analyses of an intensified regimen containing rifampicin and moxifloxacin for tuberculous meningitis. Int $\mathrm{J}$ Antimicrob Agents 45:496-503.

11. Tseng YT, Chuang YC, Shu CC, Hung CC, Hsu CF, Wang JY (2012) Empirical use of fluoroquinolones improves the survival of critically ill patients with tuberculosis mimicking severe pneumonia. Crit Care 16:R207.

12. Ruslami R, Ganiem AR, Dian S, Apriani L, Achmad TH, van der Ven AJ, Borm G, Aarnoutse RE, van Crevel R (2013) Intensified regimen containing rifampicin and moxifloxacin for tuberculous meningitis: an open-label, randomised controlled phase 2 trial. Lancet Infect Dis 13:2735.

13. Boeree MJ, Heinrich N, Aarnoutse R, Diacon AH, Dawson R, Rehal S, Kibiki GS, Churchyard G, Sanne I, Ntinginya NE, Minja LT, Hunt RD, Charalambous S, Hanekom M, Semvua HH, Mpagama SG, Manyama C, Mtfaya B, Reither K, Wallis RS, Venter A, Narunsky K, Mekota A, Henne S, Colbers A, van Balen GP, Gillespie SH, Phillips PPJ, Hoelscher M; 
PanACEA consortium (2017) High-dose rifampicin, moxifloxacin, and SQ109 for treating tuberculosis: a multi-arm, multi-stage randomized controlled trial. Lancet Infect Dis 17:39-49.

14. Heemskerk AD, Bang ND, Mai NT, Chau TT, Phu NH, Loc PP, Chau NV, Hien TT, Dung NH, Lan NT, Lan NH, Lan NN, Phong Ie T, Vien NN, Hien NQ, Yen NT, Ha DT, Day JN, Caws M, Merson L, Thinh TT, Wolbers M, Thwaites GE, Farrar JJ (2016) Intensified anti-TB therapy in adults with tuberculous meningitis. N Engl J Med 374:124-134.

15. Choi R, Jeong BH, Koh WJ, Lee SY (2017) Recommendations for optimizing tuberculosis treatment: Therapeutic drug monitoring, pharmacogenetics, and nutritional status considerations. Ann Lab Med 37:97-107.

Figure 1. Potential novel approach to anti-TB treatment in critically ill patients consisting of three phases of therapy.

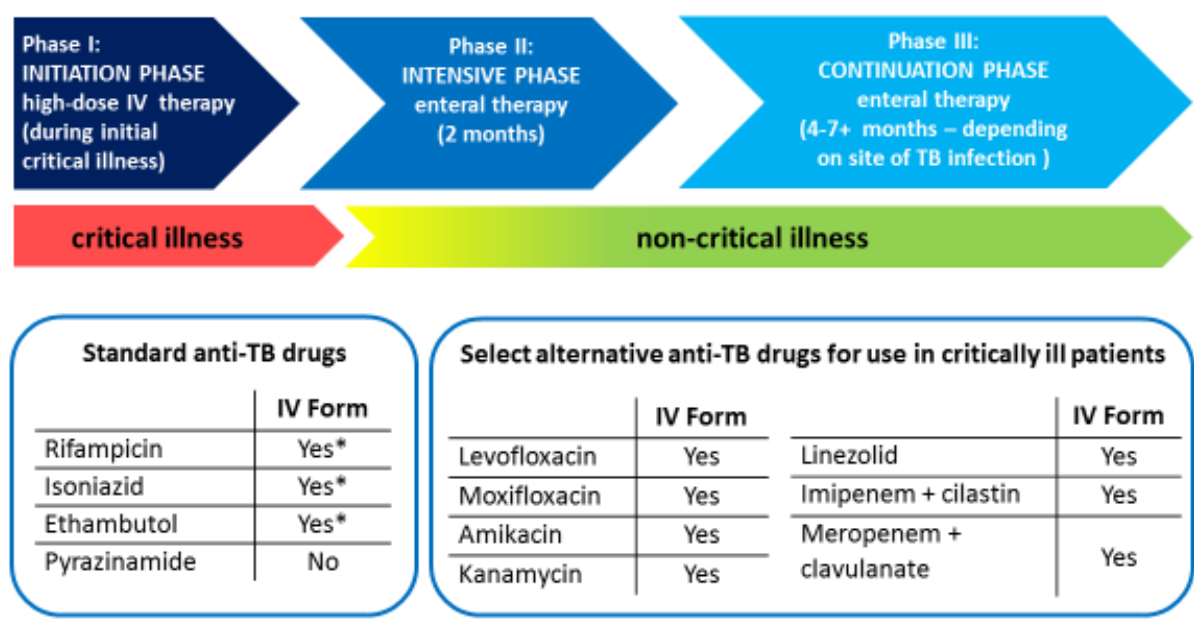

*, inconsistent availability; IV, intravenous. 